\title{
The Field-Widened SHS: An Extremely High etendue, Unscanned, Michelson-Based Spectrometer
}

\author{
John Harlander \\ Department of Physics, Astronomy and Engineering Science, St. Cloud \\ State University, St. Cloud, MN 56301 \\ F. L. Roesler and R. J. Reynolds \\ Department of Physics, University of Wisconsin-Madison, Madison, WI \\ 53706
}

\begin{abstract}
This poster described a method under development that promises to be useful for 3-D spectroscopic studies of extremely faint, spatially extended astronomical sources. The method, which we call Spatial Heterodyne Spectroscopy (SHS), is a relative of the conventional scanning Fourier Transform Spectrometer (FTS), but is free of any scanning elements. We foresee ground-based and space applications for studies of the interstellar medium and the upper atmospheres of solar system objects. Basic configurations that have been tested in the laboratory were described. The SHS instrument we are currently emphasizing for development is intended for a rocket experiment to measure C IV $\lambda 1548,51$ doublet emission at a resolving power of 20,000 from the hot ISM. Eventually we hope to provide velocity-resolved all-sky maps of selected FUV lines to complement maps obtained at other wavelengths.
\end{abstract}

\section{Introduction}

The general SHS configuration is obtained by replacing the return mirrors of a Michelson interferometer with Littrow diffraction gratings. The gratings produce a wavenumber-dependent crossing angle between monochromatic wavefronts exiting the interferometer. The resulting FIzeau fringe pattern is recorded by a position sensitive detector and the Fourier transform of this pattern recovers the spectrum. In this process, no element is mechanically scanned and the zero of the transform (zero spatial frequency in the image) corresponds to the Litrrow wavenumber of the gratings. For a more detailed description of the the basic SHS configuration see Harlander et. al. (1991).

\section{SHS Properties}

The properties of the SHS interferometer are:

- Resolving Power: Given by the limiting resolution of the grating combination. The system resolving power is given by the sum or difference 
of the individual grating resolving powers, depending upon the relative orientation of the gratings.

- Field of View: Characteristic of an interference spectrometer operating at the same resolving power (i.e. $\Omega=2 \pi / R$ where $\Omega$ is the solid angle at the gratings and $R$ is the resolving power). This is roughly two orders of magnitude larger than conventional diffraction grating instruments. In addition, field widening techniques can be applied by inserting fixed transmitting wedges into each arm of the interferometer. The resulting increase the field of view is approximately two orders of magnitude over interference spectrometers (four orders of magnitude over a grating spectrometer of equal size and resolving power).

- Spectral Range: Determined the number of pixels along one dimension of the detector. For $M$ detector elements, $M / 2$ spectral elements can be recovered without aliasing.

- Self-Scanned: The system records all spectral elements simultaneously.

- Multiplex System: Each detector element records signal from every spectral element. However, the etendue is divided among the detector elements.

- Relaxed Mechanical and Figure Tolerances: The interferometric elements are imaged onto or very near the detector. Calibration fringes monitor the alignment state and can be used to correct in software for image distortions or phase errors in the interferogram without reducing the contrast. For a description of this technique and examples of its application see Harlander et. al. (1994).

- All Reflection Configurations: All reflection SHS configurations are possible for FUV and EUV applications.

\section{References}

Douglas, N., Maaswinkel, F., Butcher, H.R. \& Frandsen, S. 1991 ESO Technical Report No. 15.

Harlander, J., 1990, Ph.D. thesis, University of Wisconsin-Madison.

Harlander, J., Roesler, F.L., Jaehnig, K., Sanders, W.T. III, \& Reynolds, R.J. 1994 in EUV, X-Ray, and Gamma-Ray Instrumentation for Astronomy $\mathrm{V}$, O. Siegmund (proc. SPIE) 2280, (in press)

Harlander, J., Roesler, F.L.,Reynolds, R.J., Jaehnig, K., \& Sanders, W. 1993 in EUV, X-Ray, and Gamma-Ray Instrumentation for Astronomy IV, 0. Siegmund (proc. SPIE) 2006, 139.

Harlander, J,. Reynolds, R.J.,\& Roesler, F.L. 1991, ApJ, 396, 730. 